\title{
Prolonged survival in advanced thymoma: Effectiveness of sequential multiple lines of chemotherapy in an inoperable case
}

\author{
MANUELA BERGONZI ${ }^{1}$, GIULIO ORLANDONI ${ }^{2}$, FRANCO CORBELLA ${ }^{3}$ and PAOLO G. GOBBI ${ }^{1}$ \\ ${ }^{1}$ Medicina Interna e Gastroenterologia, ${ }^{2}$ Cardiochirurgia, ${ }^{3}$ Radioterapia Oncologica, \\ Università di Pavia, Fondazione IRCCS Policlinico S. Matteo, Pavia, Italy
}

Received November 4, 2010; Accepted February 16, 2011

DOI: $10.3892 / \mathrm{ol} .2011 .282$

\begin{abstract}
A standard therapeutic approach for advanced malignant thymoma has yet to be defined given the rarity of this condition. We present a patient with advanced thymoma, evaluated as inoperable at diagnosis due to multiple serosal metastases. The strong constitution and determination of the patient allowed treatment with six distinct and subsequent chemotherapy regimens, all administered on an outpatient basis. A survival of 64 months from diagnosis was achieved. A favorable clinical response was obtained after the first three treatment lines, with the disappearance of all lesions on both computed tomography and positron emission tomography (PET) images. However, this result was not confirmed by surgical exploration of the thorax, undertaken with the aim of radical excision of possible residual disease. The presence of multiple pleural nodules, not evident on the imaging techniques, prevented even limited tumor debulking. The chemotherapy lines administered following detection of the lessions, stabilized the disease for a further 2 years, while a satisfactory quality of life was maintained. Only in the last months did the tumor progress and signs of cardiotoxicity appear, with the latter constituting the eventual cause of death. This case is important since the medical literature does not indicate non-cross-resistant regimens for advanced thymoma following second-line chemotherapy, and the sequence of regimens presented in this case study may serve as a feasible outline program. Moreover, we highlight the known possibility of false-negative PET studies, which can occur despite the claimed glucose avidity of thymoma tissue.
\end{abstract}

\section{Introduction}

Although thymomas are the most common tumor of the anterior mediastinum (accounting for approximately $50 \%$ of

Correspondence to: Professor Paolo G. Gobbi, Medicina Interna e Gastroenterologia, Università di Pavia, Fondazione IRCCS Policlinico S. Matteo, P.le Golgi 2, 27100 Pavia, Italy

E-mail: gobbipg@smatteo.pv.it

Key words: thymoma, 18-fluoro-deoxyglucose positron emission tomography, chemotherapy tumors in this site), their absolute frequency is extremely low. Thymomas arise from the epithelial cells of the thymus gland, show marked pathologic polymorphism and are associated with a variable prognosis, ranging from an indolent non-invasive course to aggressive behavior, with marked capacity for infiltration and metastasis (1). When presenting at an early stage, the tumor is generally capsulated and radical resection is, therefore, the cornerstone of the clinical approach in these cases. Advanced-stage disease is less commonly presented and is a well-recognized strong risk factor for both local recurrence and generalized spread of the disease. Advanced-stage disease is almost impossible to manage with radical surgery. The rarity of this presentation has been an obstacle to the development of randomized trials or, at least, of observational studies with sufficient numbers of patients. Consequently, a standard therapeutic approach for advanced thymoma has yet to be defined. The case of a patient with advanced thymoma, inoperable at diagnosis, for whom a sequence of various chemotherapy regimens obtained a satisfactory long-term clinical response while preserving a satisfactory quality of life is reported.

\section{Case report}

The patient, a 69-year-old Caucasian male, was a resident of Reggio Calabria from birth. He was healthy until September 2003, when he began to complain of cough and dysphonia. In November 2003, following certain ineffective symptomatic anti-inflammatory treatments, a chest X-ray showed enlargement of the upper mediastinum. Computed tomography (CT) of the thorax confirmed the presence of a mediastinal mass, with dimensions 8.5 wide, 7.0 high and $6.5 \mathrm{~cm}$ deep. CT showed a prevailing growth on the left in contact with the aortic arch, infiltration of the pleura and limited involvement of the pericardial sheet.

A CT-guided fine needle biopsy of the mass led to the diagnosis of thymoma B2 (WHO 2000; Table I), with histological stage IVa being determined according to the Masaoka staging criteria (Table II) (2). Due to the disease presentation, the tumor was judged to be inoperable but, due to the patient's optimal performance status and the fact that all organ function tests were normal, a relatively aggressive chemotherapy approach was planned with a possible neo-adjuvant purpose. This treatment was planned with the aim of shrinking the tumor bulk and infiltration of the contiguous viscera in order 
Table I. The WHO histological classification of thymic neoplasms (2).

\begin{tabular}{ll}
\hline Type & \multicolumn{1}{c}{ Carcinoma } \\
\hline A & Spindle cell thymoma; medullary thymoma \\
AB & Mixed thymoma \\
B1 & Lymphocyte-rich thymoma; lymphocytic thymoma; predominantly cortical thymoma \\
B2 & Cortical thymoma \\
B3 & Well-differentiated thymic carcinoma; epithelial thymoma; squamoid thymoma \\
C & Thymic carcinoma \\
\hline
\end{tabular}

Table II. Clinical staging criteria of thymoma according to Masaoka (2).

\begin{tabular}{ll}
\hline Stage & \multicolumn{1}{c}{ Criteria } \\
\hline I & Macroscopically encapsulated tumor with no microscopic capsular invasion \\
II & Macroscopic invasion surrounding fatty tissue or mediastinal pleura; \\
& microscopic invasion into the capsule \\
III & Macroscopic invasion into neighboring organs \\
IVa & Pleural or pericardial metastases \\
IVb & Lymphogenous or hematogenous metastasis
\end{tabular}

to make radical resection or, alternatively, radiotherapy technically feasible.

In March 2004, the patient, whose body surface area was $1.69 \mathrm{~m}^{2}$, underwent combination chemotherapy comprising six cycles of cisplatin $(80 \mathrm{mg})$, doxorubicin $(80 \mathrm{mg}$ ) and cyclophosphamide $(800 \mathrm{mg})$, all administered intravenously (i.v.) on day 1 of each cycle, with the cycle repeated at 21-day intervals (3). The clinical response, evaluated through a CT performed in September 2004, was evaluated as partial, but was not sufficient to allow management with local therapies.

Excellent tolerance to the first-line chemotherapy led to a second chemotherapy regimen pursuing the same neo-adjuvant aim. The new regimen, initiated in September 2004, consisted of six cycles of ifosfamide (1500 mg) i.v. on day 1, carboplatin (150 mg) i.v. on day 1 and etoposide (150 mg) i.v. on days 1-3 of each cycle, with the cycles repeated every 21 days (4). This second-line treatment was completed in January 2005, and a subsequent CT demonstrated further minor, but clear reductions, in the dimensions of the neoplastic mass.

The persistent chemosensitivity of the tumor, the limited and tolerable toxicity experienced by this elderly patient and his uncommon fighting spirit led to a third line of non-crossresistant chemotherapy, once again with the aim of making surgery or radiotherapy feasible. Six cycles of a new multipledrug combination were administered between January and June 2005. The drugs utilized were cisplatin (75 mg), epirubicin (100 mg), and etoposide (120 mg), all infused i.v. on day 1 every 21 days. CT showed a further reduction in the size of the tumor, within which fibrotic tissue prevailed, while both the hilo-mediastinal lymphadenopathies, and the pleural and pericardial infiltration showed a marked decrease.

These findings were confirmed by positron emission tomography with 18-fluoro-deoxyglucose (FDG-PET), performed in September 2005 to achieve a more accurate and sensitive assessment of the clinical response. No focal pathological uptake of the radioactive glucose was recorded throughout the body.

On the basis of these findings, the patient underwent surgical thoracotomy in January 2006 for the purposes of thorough exploration of the cavity and to remove residual tumor lesions possibly undetected by instrumental examinations. However, during surgery, a number of small pleural nodules (with diameters ranging from 6 to $12 \mathrm{~mm}$ ) were found; these nodules, which were evidence of unexpected neoplastic spread, had been completely missed on either the CT or PET images. Histology confirmed that the nodules were metastases of the malignant thymoma. The surgery was interrupted since the clinical scenario indicated that radical excision was not feasible. The intention of local radiotherapy also had to be abandoned.

The patient, therefore, received a new course of chemotherapy. Six cycles of a single-drug schedule, utilizing docetaxel at a dose of $90 \mathrm{mg}$ i.v. every 3 weeks, were administered from March to June 2006. Subsequently, liposomal doxorubicin (Myocet ${ }^{\circledR}$ ) and cisplatin at doses of 50 and $60 \mathrm{mg}$, respectively, were administered i.v. every 3 weeks for a total of eight courses, from October 2006 to May 2007 (5). Finally, the patient was treated with eight courses of vinorelbine $(50 \mathrm{mg})$, methotrexate $(100 \mathrm{mg})$ and mitoxantrone $(16 \mathrm{mg})$, administered i.v. every 3-4 weeks, between October 2007 to March 2008. The schedules of all of the chemotherapy regimens administered are shown in Table III.

Despite the subjective well-being of the patient following the completion of this last course of chemotherapy, a new CT of the thorax and abdomen showed numerous enlarged lymph nodes (with diameters from 7 to $25 \mathrm{~mm}$ ) in both the mediastinum and pulmonary hila. Shortly afterwards the patient began to complain of fatigue and increasing exhaustion, 
Table III. Drug doses and time schedules of the six chemotherapy regimens administered between March 2004 and March 2008.

\begin{tabular}{|c|c|c|c|}
\hline Drugs & Dose $\left(\mathrm{mg} / \mathrm{m}^{2}\right)$ & Route & Days \\
\hline \multicolumn{4}{|l|}{ PAC $(6 \text { cycles })^{\mathrm{a}}$} \\
\hline Cisplatin & 80 & i.v. & 1 \\
\hline Doxorubicin & 80 & i.v. & 1 \\
\hline Cyclophosphamide & 800 & i.v. & 1 \\
\hline \multicolumn{4}{|l|}{$\operatorname{ICE}(6 \text { cycles })^{\mathrm{a}}$} \\
\hline Ifosfamide & 1500 & i.v. & 1 \\
\hline Carboplatin & 150 & i.v. & 1 \\
\hline Etoposide & 150 & i.v. & $1-3$ \\
\hline \multicolumn{4}{|l|}{$\operatorname{PEV}(6 \text { cycles })^{\mathrm{a}}$} \\
\hline Cisplatin & 75 & i.v. & 1 \\
\hline Epirubicin & 100 & i.v. & 1 \\
\hline Etoposide & 120 & i.v. & 1 \\
\hline \multicolumn{4}{|l|}{ Docetaxel $(6 \text { cycles })^{b}$} \\
\hline Docetaxel & 90 & i.v. & 1 \\
\hline \multicolumn{4}{|l|}{ P-DOX-SL $(8 \text { cycles })^{b}$} \\
\hline Liposomal doxorubicin ${ }^{\mathrm{c}}$ & 50 & i.v. & 1 \\
\hline Cisplatin & 60 & i.v. & 1 \\
\hline \multicolumn{4}{|l|}{ VMN $(8 \text { cycles })^{b}$} \\
\hline Vinorelbine & 50 & i.v. & 1 \\
\hline Methotrexate & 100 & i.v. & 1 \\
\hline Mitoxantrone $^{\mathrm{a}}$ & 16 & i.v. & 1 \\
\hline
\end{tabular}

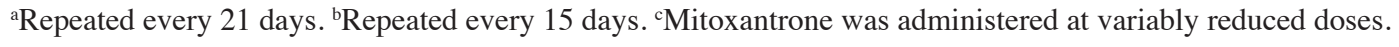

peripheral edema and breathing discomfort during exercise. The patient experienced paroxysmal nocturnal dyspnea as expression of progressive heart failure. An echocardiogram revealed impaired left ventricular function (ejection fraction $40 \%$ ) and, more importantly, a hypokinetic portion of the inferior interventricular septum with complete akinesis of the posterolateral inferior wall of the left ventricle. The patient's clinical condition deteriorated over a few weeks with the development of acute renal failure. He succumbed in April 2009 due to congestive heart failure.

\section{Discussion}

As reported in the medical literature, surgical treatment, particularly radical resection, is the optimal therapeutic approach to early-stage thymoma (at stages I, IIA and IIB). In these cases, radical surgery plays a key role in determining the best response, overcoming the importance of some aspects of disease presentation, histological type and radiotherapy. The 5-year survival of patients with encapsulated early-stage thymoma is almost $80 \%$, whereas that of patients with invasive advanced tumor is only $23 \%(6,7)$. However, the rarity of advanced presentation has prevented the collection of reliable data on the therapy and prognosis of a large series of patients. Clinical experience has evolved on the basis of anecdotal case reports or analysis of a small cohort of patients from individual institutions.
The most notable aspect of our patient's course was his favorable and stable response to chemotherapy alone (with a survival of 64 months from diagnosis), in spite of the severe initial clinical presentation (stage IVa, advanced age). Three factors can explain the response to chemotherapy. First, the determination of the patient to undergo any treatment with reasonable efficacy. Second, the long-lasting chemosensitivity of the tumor combined with the patient's good tolerance of the side effects of the six chemotherapy regimens administered. Third, the long-pursued aim of neoadjuvant efficacy of the chemotherapy, which led the clinicians to a rigorous and relatively aggressive management of drug doses, intervals of administration, and use of growth factors when required. This policy culminated in the apparently complete disappearance of the tumor on CT and PET images, justifying the subsequent surgical exploration. Although the thoracotomy revealed that the response had been incomplete, it nevertheless provided evidence of a favorable partial result that probably contributed to the prolonged survival. The false-negative CT was not completely unexpected, given the similar radiological density of both thymoma nodules and pleura. The failure of PET to reveal metastases was, however, less expected, since the utility of this imaging technique in the staging of malignant thymoma has been confirmed by the literature. PET is recommended for the detection of thoracic lesions derived from malignant thymomas, due to the extreme avidity of these lesions for fluoro- 
deoxyglucose (8). The sensitivity of the imaging technique can be further increased by the use of integrated PET/CT equipment (9).

It is likely that the number of anthracycline compounds administered, providing a cumulative dose of $651 \mathrm{mg} / \mathrm{m}^{2}$ of doxorubicin equivalents throughout the course of the disease, was responsible for the patient's heart failure (10), although only $121 \mathrm{mg} / \mathrm{m}^{2}$ was administered as compounds with minimal toxicity after the first two regimens.

In conclusion, an aggressive chemotherapy approach can be effective in advanced-stage thymoma when other treatments are not feasible. Anthracyclines and their analogues play a pivotal role in the treatment of thymoma, despite the wellrecognized risk of cardiomyopathy with refractory congestive heart failure. In our case, this complication occurred following the sixth line of chemotherapy in spite of the administration of only analogues with minimal cardiotoxicity after the first two regimens, in which a cumulative dose of $530 \mathrm{mg} / \mathrm{m}^{2}$ doxorubicin equivalents was delivered. It should be noted that both CT and PET failed to detect metastases of a thymoma, particularly when present in the form of small pleural nodules.

\section{Acknowledgements}

We are indebted to Dr Rachel Stenner for the English language revision of the manuscript. This study was supported, in part, by grants from the Fondazione IRCCS Policlinico S. Matteo, Pavia, and the 'Ferrata-Storti Foundation', Pavia.

\section{References}

1. Venuta F, Rendina EA and Coloni GF: Multimodality treatment of thymic tumors. Thorac Surg Clin 19: 71-81, 2009.

2. Kondo K: Optimal therapy for thymoma. J Med Invest 55: 17-28, 2008.

3. Kim ES, Putnam JB, Komaki R, et al: Phase II study of a multidisciplinary approach with induction chemotherapy, followed by surgical resection, radiation therapy, and consolidation chemotherapy for unresectable malignant thymomas: final report. Lung Cancer 44: 369-379, 2004.

4. Loehrer PJ Sr, Joroutek M, Aisner A, Green M, Thomas CR Jr, Livingston R and Johnson DH: Combined etoposide, ifosfamide, and cisplatin in the treatment of patients with advanced thymoma and thymic carcinoma. Cancer 91: 2010-2015, 2001.

5. Ogury T, Achiwa H, Kato D, Niimi T, Sato S and Ueda R: Efficacy of docetaxel as a second-line chemotherapy for thymic carcinoma. Chemotherapy 50: 279-282, 2004.

6. Wick MR: Prognostic factors for thymic epithelial neoplasms, with emphasis on tumor staging. Hematol Oncol Clin N Am 22: 527-542,2008.

7. Rea F, Marulli G, Giraldi R, Bortolotti L, Favaretto A Galligioni A and Sartori F: Long-term survival and prognostic factors in thymic epithelial tumors. Eur J Chardiothorac Surg 26: 412-418, 2004.

8. Quint LE: PET: other thoracic malignancies. Cancer Imaging 6: S82-S88, 2006

9. Sung YM, Lee KS, Kim BT, Choi JY, Shim YM and Yi CA: 18F-FDG PET/CT of thymic epithelial tumors: usefulness for distinguishing and staging tumor subgroups. J Nucl Med 47: 1628-1634, 2006.

10. Minotti G, Menna P, Salvatorelli E, Cairo G and Gianni L: Anthracyclines: molecular advances and pharmacologic developments in antitumor activity and cardiotoxicity. Pharmacol Rev 56: 185-229, 2004. 